\title{
Photothermal Therapy Using Gold Nanorods and Near-Infrared Light in a Murine Melanoma Model Increases Survival and Decreases Tumor Volume
}

\author{
Mary K. Popp, Imane Oubou, Colin Shepherd, \\ Zachary Nager, Courtney Anderson, and Len Pagliaro
}

Siva Therapeutics Inc, 5541 Central Avenue, Suite 140, Boulder, CO 80301, USA

Correspondence should be addressed to Len Pagliaro; len.pagliaro@sivatherapeutics.com

Received 2 June 2014; Accepted 30 July 2014; Published 21 August 2014

Academic Editor: Miguel A. Correa-Duarte

Copyright (C) 2014 Mary K. Popp et al. This is an open access article distributed under the Creative Commons Attribution License, which permits unrestricted use, distribution, and reproduction in any medium, provided the original work is properly cited.

\begin{abstract}
Photothermal therapy (PTT) treatments have shown strong potential in treating tumors through their ability to target destructive heat preferentially to tumor regions. In this paper we demonstrate that PTT in a murine melanoma model using gold nanorods (GNRs) and near-infrared (NIR) light decreases tumor volume and increases animal survival to an extent that is comparable to the current generation of melanoma drugs. GNRs, in particular, have shown a strong ability to reach ablative temperatures quickly in tumors when exposed to NIR light. The current research tests the efficacy of GNRs PTT in a difficult and fast growing murine melanoma model using a NIR light-emitting diode (LED) light source. LED light sources in the NIR spectrum could provide a safer and more practical approach to photothermal therapy than lasers. We also show that the LED light source can effectively and quickly heat in vitro and in vivo models to ablative temperatures when combined with GNRs. We anticipate that this approach could have significant implications for human cancer therapy.
\end{abstract}

\section{Introduction}

Melanoma continues to be a significant public health problem with increasing incidence and lethality [1]. In addition to surgery, chemotherapy, and radiation therapy, newer therapies such as immunotherapy [2], new generation drug treatments [3], and targeted photothermal ablation therapy [4] have been investigated for treatment of melanoma. Specifically, ablative PTT that utilizes NIR light to excite the nanoparticles offers a potentially effective tumor eradication therapy [5-8], while limiting damage to surrounding tissues [9-11].

GNRs have been shown to be the most efficient nanoparticles at absorbing NIR light and converting that energy to heat [12] and could be at least $6 \mathrm{x}$ more efficient at this task than gold nanospheres or nanoshells $[13,14]$. Coating the GNRs with amphiphilic polymer-polyethylene glycol (PEG) increases their stability and biocompatibility and enables their effectiveness for cancer therapy $[15,16]$ (Figures 1(a) and 1(b)). Nonfunctionalized PEGylated GNRs administered intravenously cannot penetrate normal blood vessels because of the tight endothelial junctions; therefore, their concentration builds up in the plasma. Over time, their concentration in solid tumors will build up, reaching several folds higher than that in plasma due to the "leaky" endothelial lining of tumor vasculature and the lack of efficient lymphatic drainage in tumors. This phenomenon is known as the enhanced permeability and retention (EPR) effect and has been observed to be widespread in solid tumors [17-20]. Functionalizing PEGylated GNRs with targeting ligands has yet to show concrete evidence of efficacy and there is some evidence that targeting ligands do not work in all cases [21, 22]. Therefore, the EPR effect may be adequate to localize PEG-coated GNRs in vascularized tumors for effective PTT [23].

Photothermal ablation efficacy of GNRs can be increased by amplifying the intensity and duration of NIR light exposure [24]. Lasers are difficult and expensive to operate and by design do not have the capabilities or practicalities of LED lights. A new generation of LED light sources, by contrast, is effective at delivering NIR light in increased intensity 


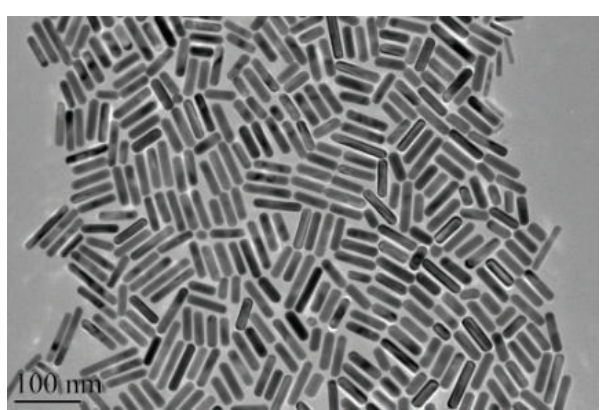

(a)

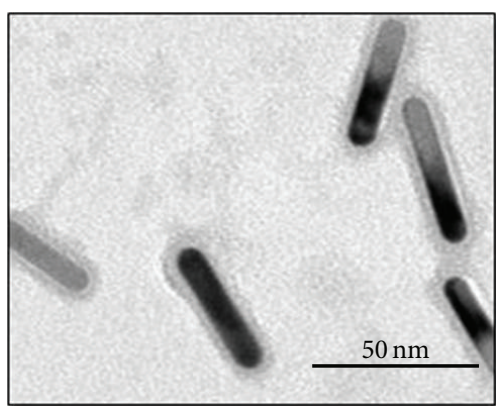

(b)

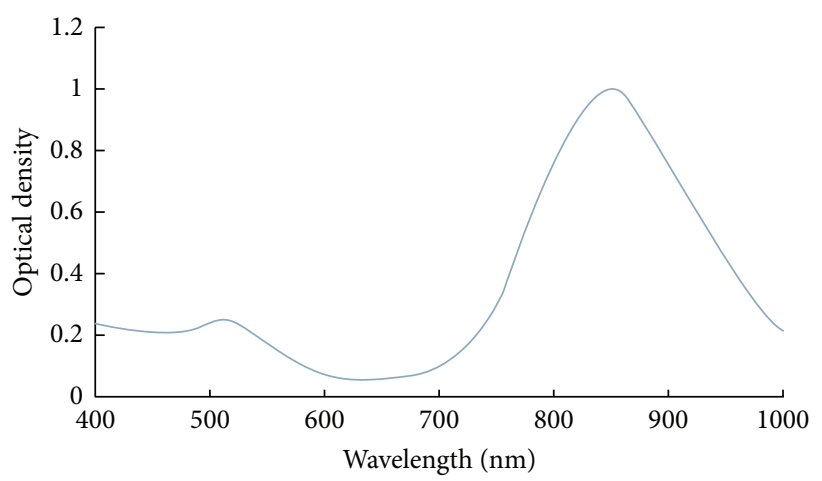

(c)

FIGURE 1: Transmission electron microscopy (TEM) analysis of GNRs before and after PEGylation and UV-Vis spectra of PEGylated GNRS. The GNRs are tuned to absorb NIR light by altering length to width ratios and the absorbed light is emitted as heat. (a) TEM image of GNRs before the addition of PEG. These GNRs are coated with CTAB. (b) TEM image of GNRs after PEGylation, with phosphotungstic acid staining to show PEG bilayer. (c) UV-Vis absorbance spectra for PEGylated GNRs, showing the LSPR at $850 \mathrm{~nm}$.

and duration while minimizing the dangers associated with lasers [25]. Compact LED light source arrays can be designed to illuminate a larger treatment area than lasers which increases the probabilities of effective use in the clinic. In this study, we use an LED NIR light source to excite GNRs in murine melanoma models as an effective cancer therapy. This research builds on previous work in the field $[9,26]$ by using an LED light source array to provide the necessary NIR light, as opposed to a conventional laser device.

\section{Materials and Methods}

2.1. Synthesis of Gold Nanorods. Gold nanorods were supplied by NanoRods LLC (Rockville, MD) synthesized with an aspect ratio for peak absorbance at $850 \mathrm{~nm}$ (Figure 1(c)). The seed-mediated method was used to synthesize the gold nanorods [26]. This method uses cetyltrimethylammonium bromide (CTAB) as the templating surfactant for synthesis. A seed solution was prepared by adding $5 \mathrm{~mL}$ of $0.2 \mathrm{M}$ CTAB to $5 \mathrm{~mL}$ of $0.0005 \mathrm{M}$ auric acid $\mathrm{HAuCl}_{4} \cdot 3 \mathrm{H}_{2} \mathrm{O}$ and reduced by the addition of $0.6 \mathrm{~mL}$ iced-cooled water and $0.01 \mathrm{M}$ sodium borohydride. The growth solution consisted of $5 \mathrm{~mL}$ of $0.001 \mathrm{M}$ auric acid added to $5 \mathrm{~mL}$ of $0.2 \mathrm{M}$ CTAB containing $250 \mu \mathrm{L}$ of $0.004 \mathrm{M}$ silver nitrate and reduced by adding $70 \mu \mathrm{L}$ of $0.0788 \mathrm{M} \mathrm{L}$-ascorbic acid. A portion $(12 \mu \mathrm{L})$ of the seed solution was added to the growth solution which then was stirred for four hours at $28-30^{\circ} \mathrm{C}$. By adjusting the silver nitrate concentration in the growth solution,
CTAB-GNRs were formed with the correct absorbance maxima. CTAB-GNRs were PEGylated with polyethylene glycol (PEG) by standard thiol PEGylation using 5000 M.W. PEG.

2.2. Gold Nanorod Dispersion Heating. A gold nanorod dispersion with an optical density (OD) of 60 was diluted to 1 $\mathrm{OD}$ using deionized water $\left(\mathrm{DIH}_{2} \mathrm{O}\right)$. The $\mathrm{OD}$ of the given solution was determined via absorbance spectroscopy and is equivalent to the observed absorbance. The solution of GNRs was diluted to OD 1 and placed in a $1 \mathrm{~cm}$ cuvette and had absorbance spectroscopy performed on the solution. An $850 \mathrm{~nm}$ LED LumiBright LE light engine (Innovations in Optics, Woburn, MA) was placed $4 \mathrm{~cm}$ above the GNRs dispersion with a flux density of $1 \mathrm{~W} / \mathrm{cm}^{2}$ at the surface of the dispersion. Temperature was measured using a calibrated FLIR E40 thermal imaging camera (FLIR Systems, Boston, MA) over 10 minutes against a water control.

2.3. In Vitro Animal Tissue Model. A swine muscle tissue model was developed to simulate the heating of a tumor infused with GNRs. The model consisted of an animal tissue base layer of $2.5 \times 2.5 \times 1 \mathrm{~cm}$, a simulated tumor, and a tissue overlay. The simulated tumor was made by shredding $1 \mathrm{~cm}^{3}$ of animal tissue and mixing with $200 \mu \mathrm{L}$ of the appropriate GNRs OD for the experiment. The GNRs-infused tissue was then loaded into a $1 \mathrm{~cm}^{3}$ indentation in the base layer of the tissue model. An animal tissue top layer of the same length 
and width as the base layer was applied on top of the base and simulated tumor to imitate tumor depth. The simulated tumor depth was varied to $0,0.5$, and $1.0 \mathrm{~cm}$.

2.4. In Vitro Animal Tissue Model with Defined Heating. A swine muscle tissue model was used to further demonstrate the ability of GNRs and NIR light to heat along defined patterns. Animal tissue was frozen and cut into $4 \times 4 \times 1 \mathrm{~cm}$ base layers, with top layers of the same dimensions. The top layer thickness was varied from $0.5 \mathrm{~cm}$ to $1 \mathrm{~cm}$. " $\mathrm{X}$ " shapes (3.5 $\mathrm{cm}$ across, $0.25 \mathrm{~cm}$ wide, $0.25 \mathrm{~cm}$ deep) were carved into the top surface of base pieces, and all tissues were thawed completely. X shapes were filled with OD $3.3(0 \mathrm{~cm}$ depth models), OD 17 (0.5 cm depth models), or OD $20(1.0 \mathrm{~cm}$ depth models) GNRs mixed with shredded tissue to simulate GNR-infused tumor tissue.

2.5. In Vitro Agar Gel Model with Defined Heating. Agar gel was melted and molded into round base pieces approximately $2.0 \mathrm{~cm}$ in thickness and $5.5 \mathrm{~cm}$ in diameter. " $\mathrm{X}$ " shapes $(\sim 5 \mathrm{~cm}$ across, $0.25 \mathrm{~cm}$ wide, $0.25 \mathrm{~cm}$ deep) were carved into the top surface of base pieces and filled with OD 6 GNRs suspension mixed with agar gel to simulate GNRs-infused tumor tissue. The NIR light source was set to produce $1 \mathrm{~W} / \mathrm{cm}^{2}$ power at the tumor model level that varied from 2.5 to $4.5 \mathrm{~cm}$ above the model surface, and models were placed directly under the light for ten minutes. Tumor temperature was measured and photographed each minute during NIR light exposure using a FLIR E40 thermal camera. The OD 6 mixture was used to model defined heating shapes. Three trials were conducted for each experiment set, plus one control trial that replaced GNR suspension with distilled water.

2.6. In Vitro Photothermal Heating. The in vitro tissue model was exposed to NIR light for a total of 10 minutes via the LED light source. Temperature of the tumor was monitored every 2 minutes by lifting the top tissue layer to expose the tumor and recording a thermal image. A FLIR E40 thermal imaging camera in combination with FLIR Tools+ was used to acquire, record, and analyze thermal images. The flux density of the NIR light source was maintained in the range of 3.3-3.7 W/ $/ \mathrm{cm}^{2}$. The concentration of injected GNRs was incrementally increased until ablation temperatures were observed for each tumor depth. An average temperature over $n=3$ trials per concentration was assessed.

For the animal tissue model showing defined heating with a GNRs-infused "X", the NIR LED was set to $2.2 \mathrm{~W} / \mathrm{cm}^{2}$ flux density at the GNRs level. Models were placed $2 \mathrm{~cm}$ under the light source. $N=3$ trials were conducted in each tumor depth, with one additional control trial replacing GNRs suspension with distilled water. Top layers $(0.5$ or $1 \mathrm{~cm}$ thick) were placed on top of the bases during those models' light exposure periods. Temperatures at both top surface and tumor level were measured and photographed every 30 seconds using a FLIR E40 thermal camera. Models were removed when the tumor temperature reached approximately $55^{\circ} \mathrm{C}$.
2.7. Initial GNRs PTT Efficacy Study in a Mouse Melanoma Model. All murine animal studies were performed at Bolder BioPATH Inc. (Boulder, CO). Mouse melanoma cells from the B16F10 line were cultured and implanted into female C57BL/6 mice, weighing between 18 and $21 \mathrm{~g}$ (Harlan Inc., Indianapolis, IN) [27]. The B16F10 tumor cells were obtained from the DCT Tumor Repository (Frederick, MD, May 17, 1991); the cells were then tested via the MAP method on November 6, 1991, with negative results for bacteria, fungi, and mycoplasma. The cells were maintained in LN2 storage until being thawed and expanded in culture. The tumors were allowed to grow 7-10 days to a uniform size across all treatment groups. There were four treatment groups, each with $n=6:$ GNRs + NIR light, GNRs + no light, saline + NIR light, and saline + no light. All procedures were performed within IACUC ethical standards and practices.

After the tumors reached a uniform size, the treatment groups were injected with either $200 \mu \mathrm{L}$ OD 60 PEG-GNRs (approximately $14 \mathrm{mg}$ gold per kg body weight) or $200 \mu \mathrm{L}$ sterile saline (control). The GNRs were then allowed to circulate within the body for 48 hrs before exposure to the NIR light. After being anesthetized with isoflurane, each mouse was exposed to NIR light for a total of $6.5 \mathrm{~min}$, maintaining external tumor temperatures in the ablation zone $\left(55-65^{\circ} \mathrm{C}\right)$ for at least $5 \mathrm{~min}$. Temperature was monitored using a FLIR E40 thermal imaging camera in combination with FLIR Tools+. The power density of the NIR LED light was regulated to maintain a constant external tumor temperature, staying within the range of $0.25-0.50 \mathrm{~W} / \mathrm{cm}^{2}$. Tumor volume and body weight were recorded every 2-3 days for the duration of the study. The animals were sacrificed when tumor volume reached $10 \%$ of their total body weight.

Animals were initially injected with PEG-GNRs or saline on day 0 . The first NIR light treatment took place on day 2. Subsequent treatments took place on day 7 and day 15 as needed for the remaining mice. Mice from the GNRs + no light group and the saline + no light group were sacrificed on day 8. Mice from the saline + NIR light group were sacrificed on day 12. Mice from the GNRs + NIR light group survived until day 29. Treatments occurred on days 2, 7, and 15 , repeated as needed to mitigate tumor growth in the surviving mice. The initial treatment on day 2 was performed as described above. The second treatment, on day 7, consisted of 10 separate local injections of OD 60 PEG-GNRs at $10 \mu \mathrm{L}$ each, around the periphery of the tumor. The tumor was then exposed to NIR light for 6.5 minutes, at appropriate power levels to maintain ablation temperatures. The third treatment was administered as a systemic treatment, in which on day 15 each surviving mouse was injected with $200 \mu \mathrm{L}$ of OD 60 PEG-GNRs and then allowed 48 hrs for the PEG-GNRs to circulate within the body. The tumor was then exposed to NIR light for 6.5 minutes, at appropriate power levels to maintain ablation level temperatures.

\section{Results}

3.1. Initial Bench Heating Demonstration and Nontissue Models. GNRs can be highly tuned to resonate at specific wavelengths by altering their dimensions during growth. It is 


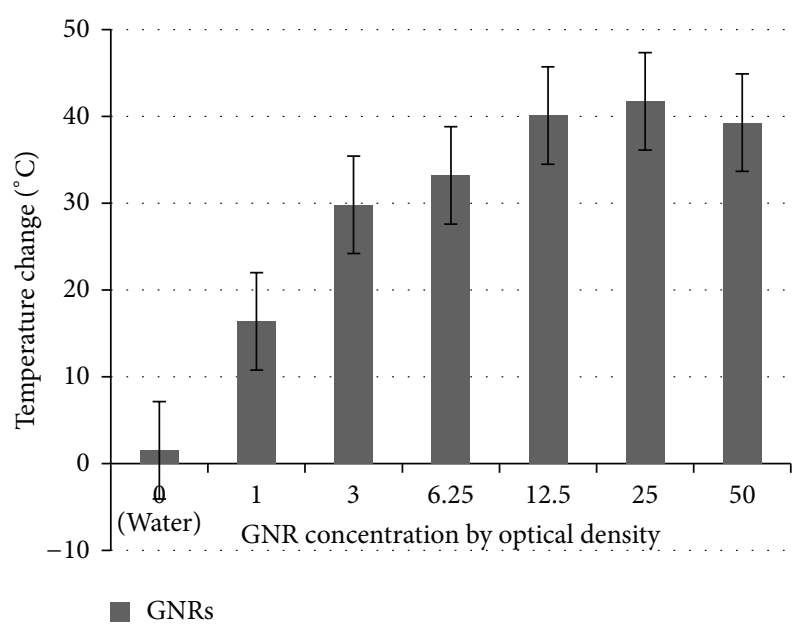

(a)

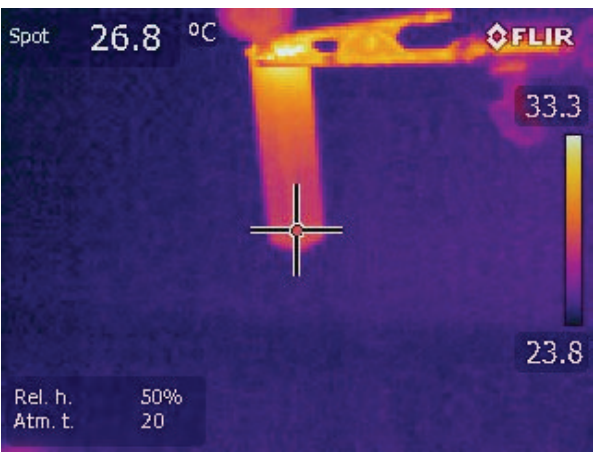

(b)

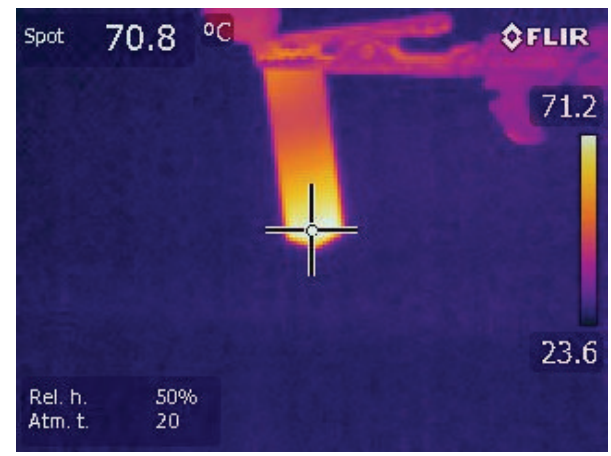

(c)

FIGURE 2: Photothermal properties of GNRs in nontissue models nanorods. (a) Chart of varying concentrations of GNRs after exposure to NIR light ( $1 \mathrm{~W} / \mathrm{cm}^{2}, n=3,1$ min exposure). (b) and (c) Thermographic images of water (b) and GNRs (OD 50, (c)) in microcentrifuge tubes after NIR exposure.

known that NIR light between $650 \mathrm{~nm}$ and $1450 \mathrm{~nm}$ can pass through tissue with minimal loss of power; therefore, GNRs for PTT in melanoma should be tuned to a wavelength in this region [28]. In this study we utilized GNRs and an LED light engine specific to $850 \mathrm{~nm}$ which is in the middle of this therapeutic window and increases NIR light penetration.

GNRs increased in temperature proportionally as the solutions increased in concentration, until approximately $\mathrm{OD}$ 12.5 , at which point the temperature delta reached a plateau (see Figure 2(a)). An identical temperature delta plateau was observed in subsequent in vitro models (not published). Figures 2(b) and 2(c) show thermal images taken by a FLIR E40 camera of a water control and OD 50 GNRs after NIR exposure $\left(1 \mathrm{~min}, 1 \mathrm{~W} / \mathrm{cm}^{2}\right)$. In the agar gel models, the temperature of the GNR filled " $\mathrm{X}$ shape" was recorded over a period of 10 minutes. The gel model was able to reach temperatures in the ablation zone, while maintaining defined distinction between the GNR gel and the non-GNR gel.

\subsection{Analysis of Treatable Tumor Depths and Heating within} Defined Patterns in Animal Tissue Models. Animal tissue models were used to show the ability of GNRs PTT to reach and hold ablation temperatures. Ablation level temperatures were observed at the tumor level for all tumor depths up to $1 \mathrm{~cm}$. For $0,0.5$, and $1 \mathrm{~cm}$ depths, GNRs ODs of 1,10 , and 12 , respectively, were necessary to reach these elevated heating levels. The majority of heating occurred in the first 4 minutes of NIR light exposure, and the observed temperature was maintained through the duration of the experiment (Figure 3(a)).

The animal tissue model was also used to demonstrate the ability of GNRs to heat along defined patterns when exposed to NIR light. Figures 3(b) and 3(c) show the difference in temperature before and after NIR exposure. An "X" shape filled with GNRs shows defined heating patterns restricted to the presence of GNRs. The tissue models were exposed to NIR light at $3.3 \mathrm{~W} / \mathrm{cm}^{2}$ for 120 seconds and then removed from light exposure once the model reached ablative temperatures. While the in vitro models we used lack perfusion, which has significant consequences for tissue heating dynamics [29], they provided a rapid, easy method of evaluating tissue heating effectiveness, and they were valuable in developing dosing guidelines for both GNRs and the NIR light.

3.3. Initial GNRs PTT Efficacy Study in a Mouse Melanoma Model. In this study, efficacy was demonstrated by exposing 


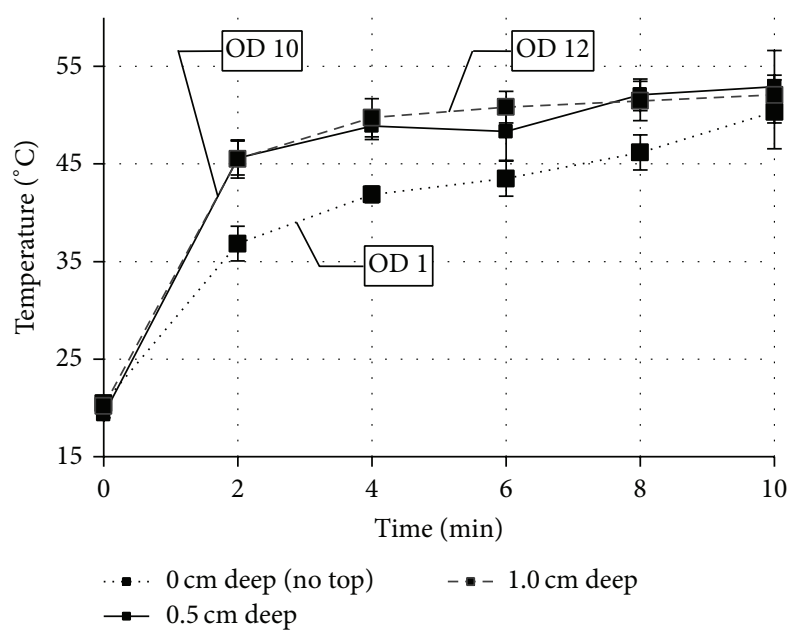

(a)

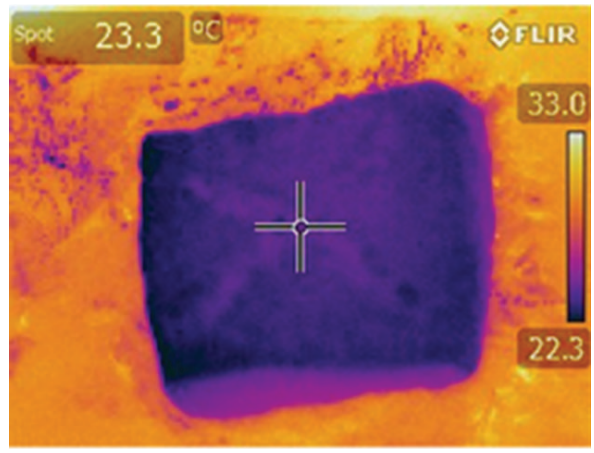

(b)

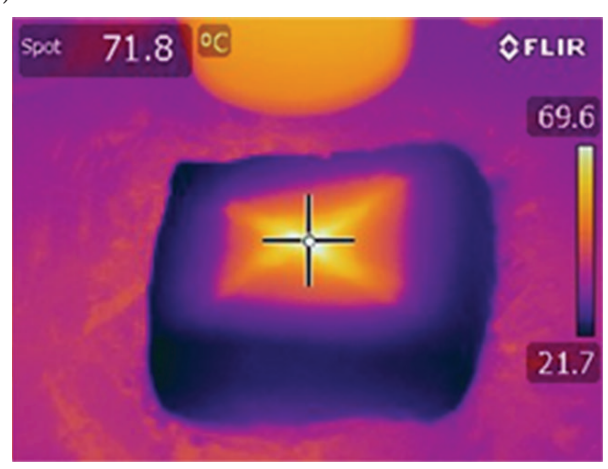

(c)

FIGURE 3: Photothermal properties of GNRs in animal tissue models. (a) Graph showing temperature increase over time for different combinations of GNR concentrations and tumor depths. OD 1 was used for superficial tumor models $(0 \mathrm{~cm}$ deep), OD 10 was used for $0.5 \mathrm{~cm}$ deep tumor models, and OD 12 was used for $1.0 \mathrm{~cm}$ deep tumor models in order to reach target ablation temperatures. All models were exposed to $3.5 \mathrm{~W} / \mathrm{cm}^{2}$ of NIR light. (b) and (c) Thermographic images of GNR filled " $\mathrm{X}$ " in animal tissue models, before ((b), $\left.t=0\right)$ and after $((\mathrm{c}), t=120 \mathrm{~s})$ NIR light exposure (OD $\left.3.3,2.2 \mathrm{~W} / \mathrm{cm}^{2}\right)$.

aggressive B16F10 murine melanoma tumors in C57 mice to PEG-GNR PTT, in which PEGylated GNRs localized to tumor tissues via the EPR effect and the applied NIR light provided energy for heating the GNRs and thus the tumors. The primary end points of this study were tumor volume and survival.

The fully treated mice (GNRs + NIR light) lived for up to 29 days after initial injection of the PEG-GNRs, whereas the saline + NIR light group survived until day 13 (Figure 4(a)). Both groups that did not receive NIR light treatment were sacrificed on day 9 due to tumor growth. This study also examined average tumor volume throughout the course of the treatment. Tumor volumes were recorded every 2-3 days for all surviving mice (Figure 4(b)). The average tumor volume of the fully treated mice (GNRs + NIR light) showed a decrease after PEG-GNRs PTT. The control groups did not show a decrease in tumor volume after treatment.

\section{Discussion}

We present the development of a novel PTT approach as a potential melanoma treatment. We demonstrate significant reduction in tumor volume and more than double animal survival compared to untreated controls. These results are very favorable compared to studies using the current generation of melanoma drugs. Until recently, published work has shown that only NIR lasers have the power needed to excite GNRs $[25,30]$. In the current research, we demonstrate that ablative temperatures can be reached using a high powered LED device. This dramatically reduces the complications of using GNRs combined with NIR light as a therapy, as NIR from an LED is safer and easier to use when compared to the use of a laser [30]. The ability of an LED device to excite the GNRs was demonstrated first using a simple GNRs suspension and then shown to be effective at reaching ablative temperatures in in vitro models.

From our in vitro experiments, we show that the LED device is able to effectively heat tumor models at varying tissue depths up to $1.5 \mathrm{~cm}$. Temperatures within the ablation range $\left(55-65^{\circ} \mathrm{C}\right)$ were met and maintained at the tumor level, by adjusting the GNRs concentration within the model and the applied flux density of the LED's power output. While the in vitro tumor models lack some features of living tissue, notably perfusion, the results of the animal experiments 


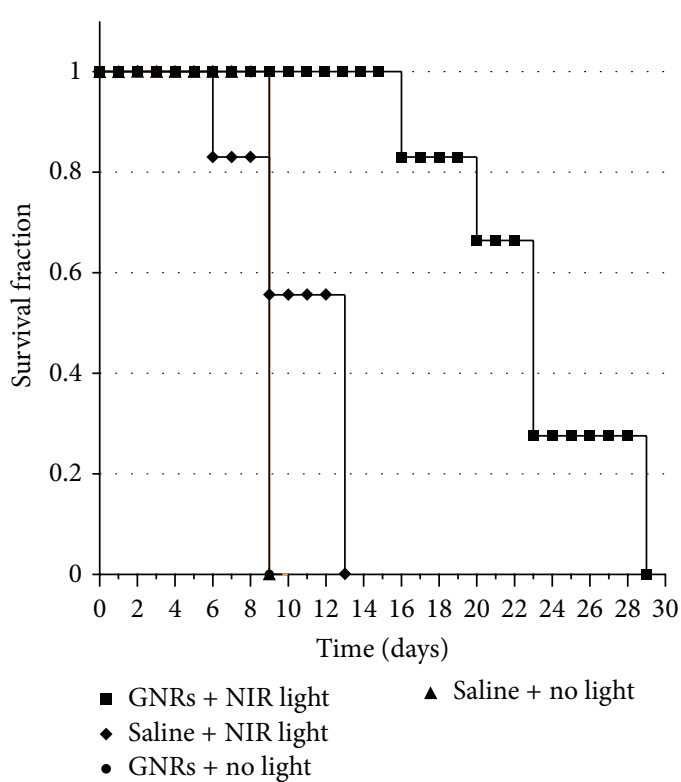

(a)

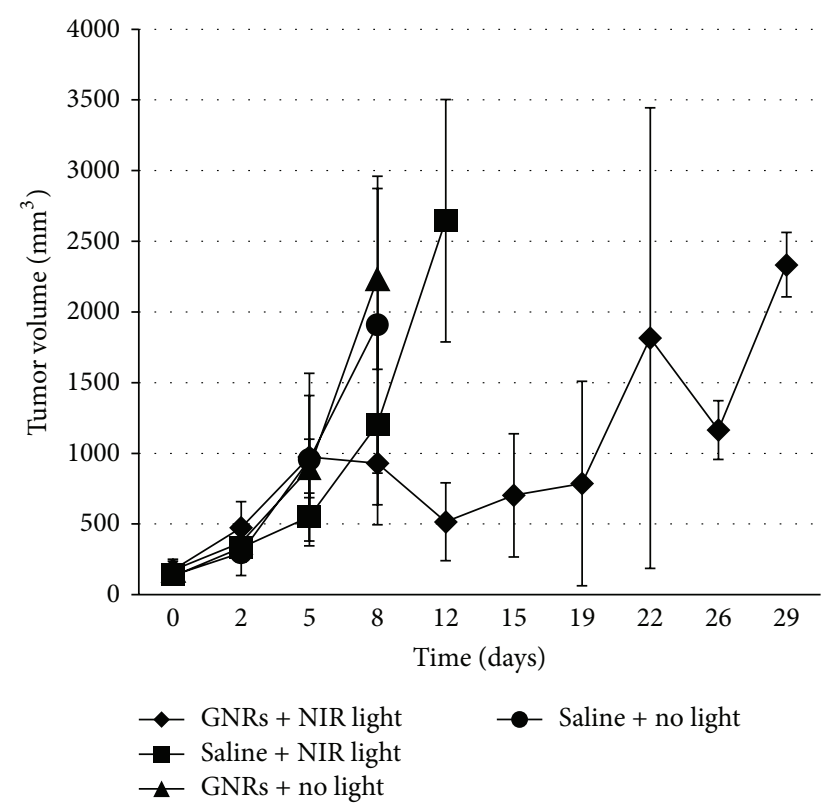

(b)

FIGURE 4: Efficacy of photothermal therapy demonstrated in the treatment of mice bearing murine melanoma tumors. C57BL/6 mice bearing F10B16 tumors were injected with either PEG-GNRs $(200 \mu \mathrm{L}$, OD60) or saline $(200 \mu \mathrm{L})$; the PEG-GNRs were then allowed 48 hrs to circulate within the body, and then half of the mice were exposed to NIR light to maintain ablation temperatures for 6 min $(n=6)$. Their overall survival and tumor volume were tracked throughout the entire length of the study (tumor measurements were taken every 2-3 days). (a) Survival of the mice is plotted against time (days), with day $=0$ signifying initial PEG-GNR/saline injection. (b) Tumor volume (mm ${ }^{3}$ ) is plotted versus time (days) after initial injection with PEG-GNRs/saline. The data in (a) and (b) were censored.

demonstrate that therapeutically effective heating is achieved in vivo. In vitro models also showed that heat is only observed in the presence of NIR exposed GNRs. This translates into localized heating of defined and predictable patterns, specific to the GNRs only; from this it is clear that illuminating healthy tissue without the presence of GNRs generates minimal heating and minimal damage.

PEG-GNRs have been shown to effectively accumulate in solid tumors due to the EPR effect [17, 31, 32]. Previously, it has been reported that GNRs without the PEG coating are unable to accumulate within solid tumors, due to their lack of biocompatibility and stability $[33,34]$. PEG provides the GNRs with an increased half-life and greater biodistribution within in vivo models $[31,35,36]$. In the current study, PEGGNRs combined with NIR light from an LED device were shown to increase animal survival and reduce overall tumor volume. Without the addition of the PEG coating to the GNRs, there would have been immediate clearance of the GNRs from the body and therefore minimal accumulation of the GNRs in the tumor, and the PTT would have been largely ineffective. It can be concluded that PEG, or a similar biocompatible polymer, is essential when conducting in vivo experiments with GNRs, in order to allow the GNRs to fully disperse within the body and allow for accumulation in tumor tissues. In addition, the PEG coating stabilized the GNRs during heating, thus keeping them from altering their morphology and heating ability. No changes in the morphology or heating ability of the PEG-coated GNRs were observed in this study.

Future studies will investigate the biodistribution and clearance of PEG-GNRs within the body, as well as the effects of higher GNRs concentration and power density for in vivo use. More detailed studies examining the dosimetry of the observed temperatures during PTT and the duration of treatment are needed [29], in order to develop a model that can be used for predicting appropriate treatment settings based on patient variables. This would aid in correlating the recorded temperatures with tumor volume reduction and increased survival. Such information is imperative in order to make this therapy an effective clinical treatment.

\section{Conflict of Interests}

The authors declare that there is no conflict of interests regarding the publication of this paper.

\section{Acknowledgments}

This work was supported in part by grants from Breakout Labs, part of the Thiel Foundation, and from the Colorado Office of Economic Development Bioscience Discovery Evaluation Grant Program. The authors thank Dr. Dave Emerson, Bolder BioPATH, for his advice and expertise on the animal models. 


\section{References}

[1] E. C. Dunki-Jacobs, G. G. Callender, and K. M. McMasters, "Current management of melanoma," Current Problems in Surgery, vol. 50, no. 8, pp. 351-382, 2013.

[2] D. Zikich, J. Schachter, and M. J. Besser, "Immunotherapy for the management of advanced melanoma: the next steps," American Journal of Clinical Dermatology, vol. 14, no. 4, pp. 261272,2013

[3] A. M. M. Eggermont and C. Robert, "New drugs in melanoma: it's a whole new world," European Journal of Cancer, vol. 47, no. 14, pp. 2150-2157, 2011.

[4] W. Lu, C. Xiong, G. Zhang et al., "Targeted photothermal ablation of murine melanomas with melanocyte-stimulating hormone analog-conjugated hollow gold nanospheres," Clinical Cancer Research, vol. 15, no. 3, pp. 876-886, 2009.

[5] L. C. Kennedy, L. R. Bickford, N. A. Lewinski et al., "A new era for cancer treatment: gold-nanoparticle-mediated thermal therapies," Small, vol. 7, no. 2, pp. 169-183, 2011.

[6] W. Cai, T. Gao, H. Hong, and J. Sun, "Applications of gold nanoparticles in cancer nanotechnology," Nanotechnology, Science and Applications, vol. 1, pp. 17-32, 2008.

[7] Y. Zhang, W. Zhang, C. Geng et al., "Thermal ablation versus conventional regional hyperthermia has greater anti-tumor activity against melanoma in mice by upregulating $\mathrm{CD} 4^{+}$cells and enhancing IL-2 secretion," Progress in Natural Science, vol. 19, no. 12, pp. 1699-1704, 2009.

[8] W. Lu, M. P. Melancon, C. Xiong et al., "Effects of photoacoustic imaging and photothermal ablation therapy mediated by targeted hollow gold nanospheres in an orthotopic mouse xenograft model of glioma," Cancer Research, vol. 71, no. 19, pp. 6116-6121, 2011.

[9] G. von Maltzahn, J. Park, A. Agrawal et al., "Computationally guided photothermal tumor therapy using long-circulating gold nanorod antennas," Cancer Research, vol. 69, no. 9, pp. 38923900, 2009.

[10] G. Von Maltzahn, A. Centrone, J. Park et al., "SERS-coded cold nanorods as a multifunctional platform for densely multiplexed near-infrared imaging and photothermal heating," Advanced Materials, vol. 21, no. 31, pp. 3175-3180, 2009.

[11] E. B. Dickerson, E. C. Dreaden, X. Huang et al., "Gold nanorod assisted near-infrared plasmonic photothermal therapy (PPTT) of squamous cell carcinoma in mice," Cancer Letters, vol. 269, no. 1, pp. 57-66, 2008.

[12] L. Tong, Q. Wei, A. Wei, and J. Cheng, "Gold nanorods as contrast agents for biological imaging: optical properties, surface conjugation and photothermal effects," Photochemistry and Photobiology, vol. 85, no. 1, pp. 21-32, 2009.

[13] J. R. Cole, N. A. Mirin, M. W. Knight, G. P. Goodrich, and N. J. Halas, "Photothermal efficiencies of nanoshells and nanorods for clinical therapeutic applications," Journal of Physical Chemistry C, vol. 113, no. 28, pp. 12090-12094, 2009.

[14] P. K. Jain, K. S. Lee, I. H. El-Sayed, and M. A. El-Sayed, "Calculated absorption and scattering properties of gold nanoparticles of different size, shape, and composition: applications in biological imaging and biomedicine," Journal of Physical Chemistry B, vol. 110, no. 14, pp. 7238-7248, 2006.

[15] A. V. Liopo, A. Conjusteau, O. V. Chumakova, S. A. Ermilov, R. Su, and A. A. Oraevsky, "Highly purified biocompatible gold nanorods for contrasted optoacoustic imaging of small animal models," Nanoscience and Nanotechnology Letters, vol. 4, no. 7, pp. 681-686, 2012.
[16] T. B. Huff, L. Tong, Y. Zhao, M. N. Hansen, J. Cheng, and A. Wei, "Hyperthermic effects of gold nanorods on tumor cells," Nanomedicine, vol. 2, no. 1, pp. 125-132, 2007.

[17] Y. Akiyama, T. Mori, Y. Katayama, and T. Niidome, "The effects of PEG grafting level and injection dose on gold nanorod biodistribution in the tumor-bearing mice," Journal of Controlled Release, vol. 139, no. 1, pp. 81-84, 2009.

[18] A. J. Gormley, N. Larson, S. Sadekar, R. Robinson, A. Ray, and H. Ghandehari, "Guided delivery of polymer therapeutics using plasmonic photothermal therapy," Nano Today, vol. 7, no. 3, pp. 158-167, 2012.

[19] M. K. Danquah, X. A. Zhang, and R. I. Mahato, "Extravasation of polymeric nanomedicines across tumor vasculature," Advanced Drug Delivery Reviews, vol. 63, no. 8, pp. 623-639, 2011.

[20] H. Maeda, J. Wu, T. Sawa, Y. Matsumura, and K. Hori, “Tumor vascular permeability and the EPR effect in macromolecular therapeutics: A review," Journal of Controlled Release, vol. 65, no. 1-2, pp. 271-284, 2000.

[21] K. F. Pirollo and E. H. Chang, "Does a targeting ligand influence nanoparticle tumor localization or uptake?" Trends in Biotechnology, vol. 26, no. 10, pp. 552-558, 2008.

[22] A. S. Mikhail and C. Allen, "Block copolymer micelles for delivery of cancer therapy: transport at the whole body, tissue and cellular levels," Journal of Controlled Release, vol. 138, no. 3, pp. 214-223, 2009.

[23] K. Greish, "Enhanced Permeability and Retention (EPR) effect for anticancer nanomedicine drug targeting," in Cancer Nanotechnology: Methods in Molecular Biology, vol. 624, chapter 3, pp. 25-38, 2010.

[24] S. Kessentini and D. Barchiesi, "Quantitative comparison of optimized nanorods, nanoshells and hollow nanospheres for photothermal therapy," Biomedical Optics Express, vol. 3, no. 3, pp. 590-604, 2012.

[25] R. Vankayala, Y. K. Huang, P. Kalluru, C. S. Chiang, and K. C. Hwang, "First demonstration of gold nanorods-mediated photodynamic therapeutic destruction of tumors via nearinfrared light activation," Small, p. 1002, 2013.

[26] X. Huang, P. K. Jain, I. H. El-Sayed, and M. A. El-Sayed, "Plasmonic photothermal therapy (PPTT) using gold nanoparticles," Lasers in Medical Science, vol. 23, no. 3, pp. 217-228, 2008.

[27] I. J. Fidler, "Selection of successive tumour lines for metastasis," Nature, vol. 242, no. 118, pp. 148-149, 1973.

[28] V. J. Pansare, S. Hejazi, W. J. Faenza, and R. K. Prud'Homme, "Review of long-wavelength optical and NIR imaging materials: contrast agents, fluorophores, and multifunctional nano carriers," Chemistry of Materials, vol. 24, no. 5, pp. 812-827, 2012.

[29] P. S. Yarmolenko, E. J. Moon, C. Landon et al., "Thresholds for thermal damage to normal tissues: an update," International Journal of Hyperthermia, vol. 27, no. 4, pp. 320-343, 2011.

[30] X. Huang, I. H. El-Sayed, W. Qian, and M. A. El-Sayed, "Cancer cell imaging and photothermal therapy in the nearinfrared region by using gold nanorods," Journal of the American Chemical Society, vol. 128, no. 6, pp. 2115-2120, 2006.

[31] J. Milton Harris, N. E. Martin, and M. Modi, "Pegylation: a novel process for modifying pharmacokinetics," Clinical Pharmacokinetics, vol. 40, no. 7, pp. 539-551, 2001.

[32] K. Lin, A. Bagley, A. Zhang, D. Karl, S. Yoon, and S. Bhatia, "Gold nanorods photothermal therapy in a genetically engineered mouse model of soft tissue sarcoma," Nano LIFE, vol. 1, no. 3-4, pp. 277-287, 2011. 
[33] J. Lipka, M. Semmler-Behnke, R. A. Sperling et al., "Biodistribution of PEG-modified gold nanoparticles following intratracheal instillation and intravenous injection," Biomaterials, vol. 31, no. 25, pp. 6574-6581, 2010.

[34] J. C. Y. Kah, K. Y. Wong, K. G. Neoh et al., "Critical parameters in the pegylation of gold nanoshells for biomedical applications: an in vitro macrophage study," Journal of Drug Targeting, vol. 17, no. 3, pp. 181-193, 2009.

[35] P. Bailon and C. Y. Won, "PEG-modified biopharmaceuticals," Expert Opinion on Drug Delivery, vol. 6, no. 1, pp. 1-16, 2009.

[36] G. F. Paciotti, L. Myer, D. Weinreich et al., "Colloidal gold: a novel nanoparticle vector for tumor directed drug delivery," Drug Delivery, vol. 11, no. 3, pp. 169-183, 2004. 

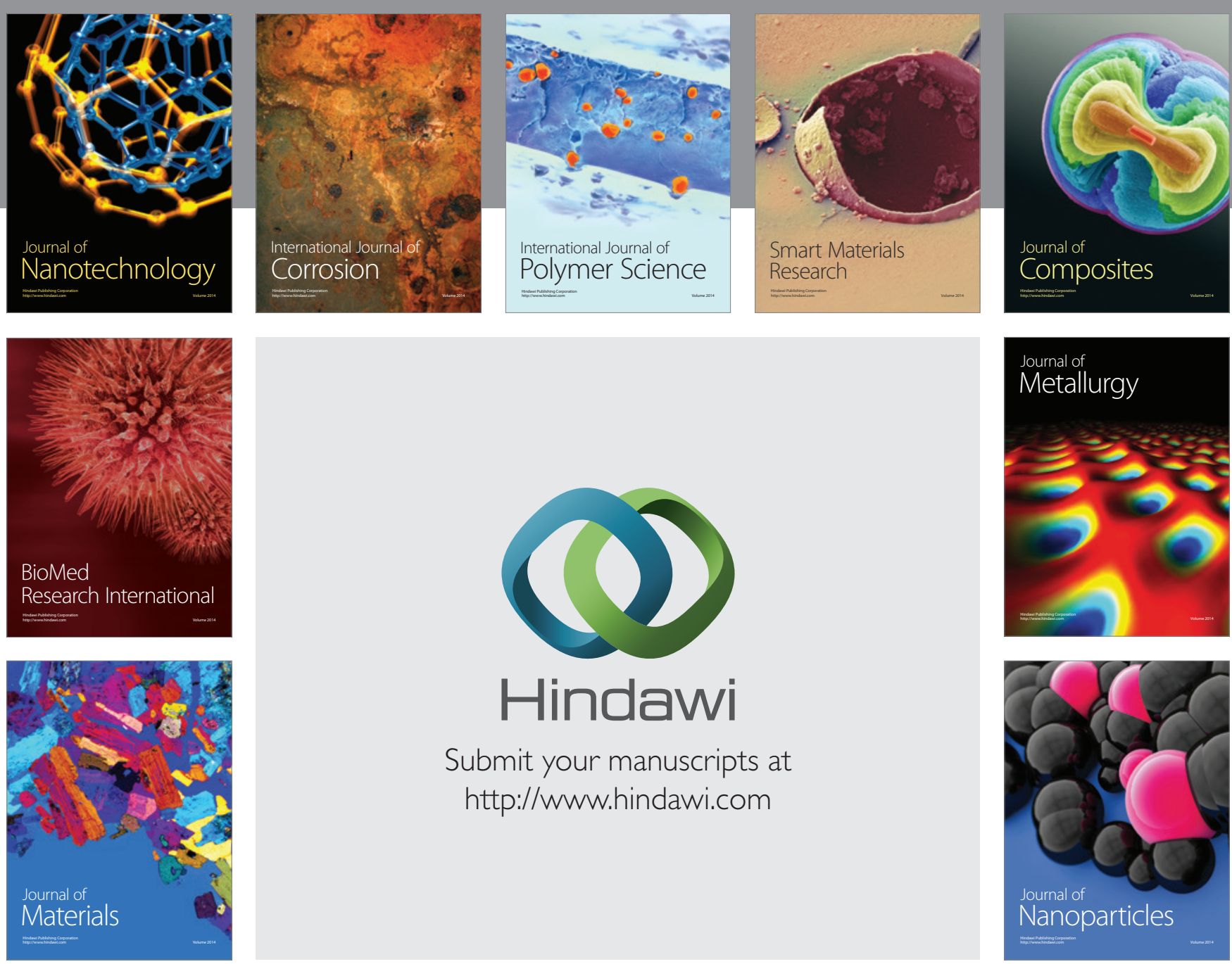

Submit your manuscripts at http://www.hindawi.com
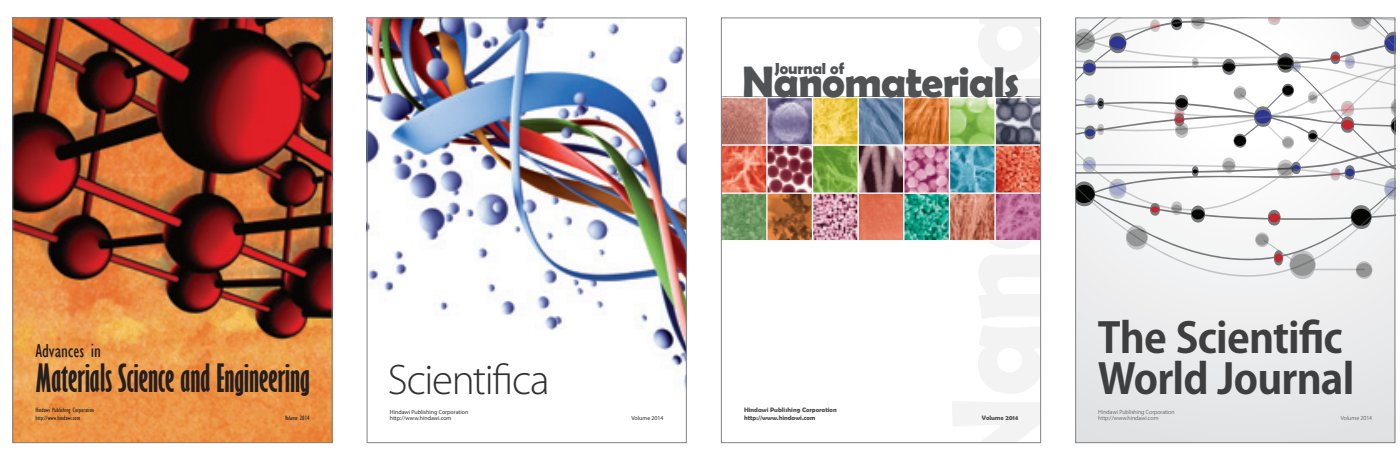

\section{The Scientific World Journal}
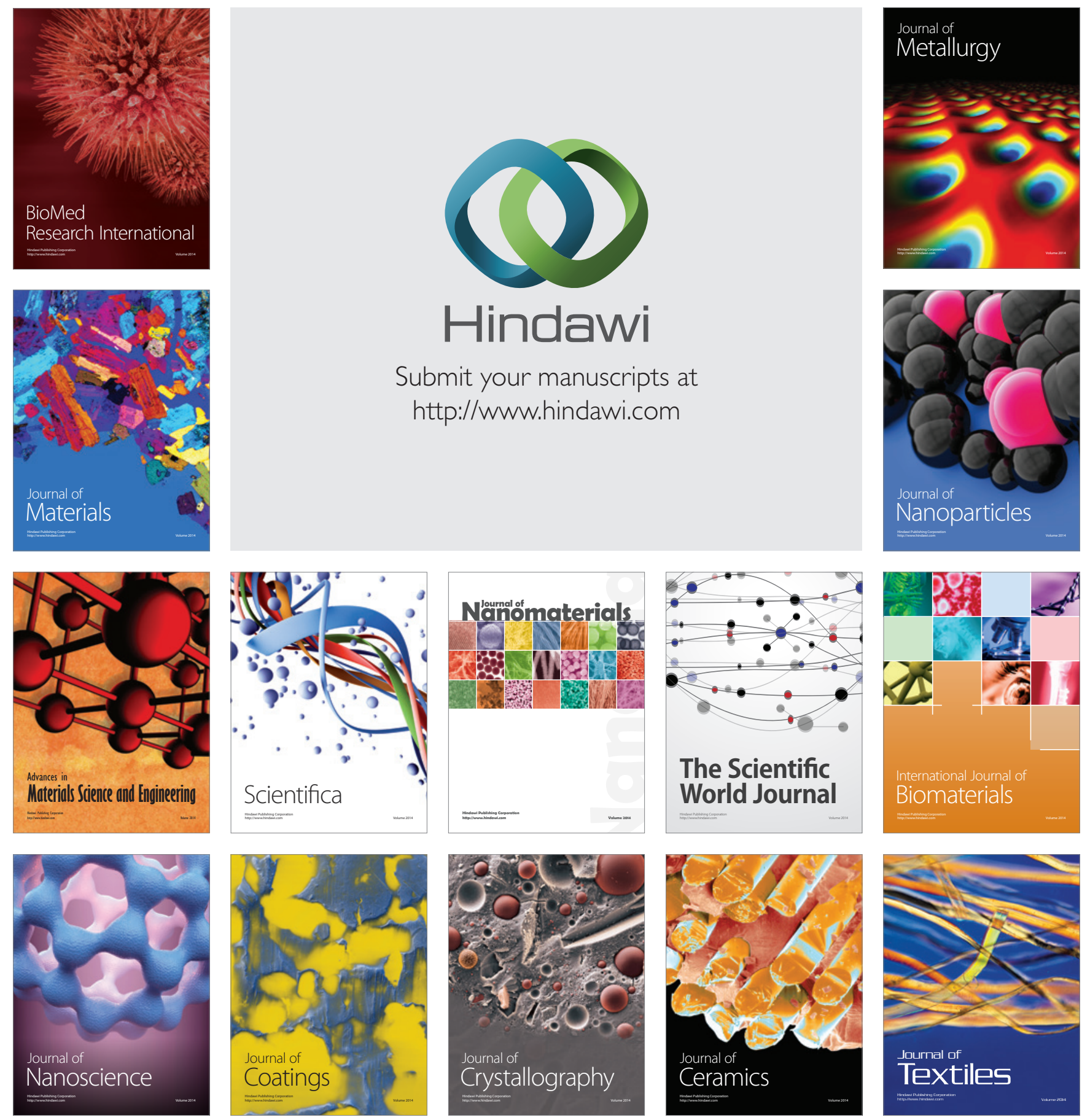